\title{
Integrating IoT and WSN for Detection \& Tracking
}

\author{
A.Y. Prabhakar, Akshit Sharma, Ashish Kumar Singh, Tushar Sharma
}

\begin{abstract}
The project throws light on detection of object and afterwards tracking of the said object using IOT and WSN. All the operations will be performed in real time as capturing of images is a continuous process which is achieved with the help of ESP32CAM mounted on the chassis of the robot and its connection is given to ESP32CAM. Ultrasonic detects object and tracking by robot is done by its right or left movements and backward or forward movements depending on the said object's displacement. The distance between the robot and the said object is constant which is preserved with the help of ultrasonic sensors. Tracking involves live video feed and trigger of manual mode for detecting the object. Once the object is detected it will be intimated through WSN to base station and through IoT local and central headquarters for further analysis.
\end{abstract}

Keywords: ESP32CAM, WSN, IoTs.

\section{INTRODUCTION}

Various industries such as defense, academic and research communities are using popular robotics products which makes the robotics field grow exponentially. The design and implementation cost of a robot is very less than hiring a human care giver. The robots can be reprogrammed faster and more efficient. A large area can be covered by a robot with sufficient intelligence. The intelligent robots can perform preferred tasks in unstructured environments with or without human direction. Safety and security can be increased using real time object detection by taking the aid of remote monitoring and control system. Basically the surveillance systems are building up with multiple cameras which are placed in different angles of view to track objects. In the present scenario tracking object requires camera which in turn increases the number of camera. In proposed system a ESP32 CAM is installed on a robot and the robot can move various directions to take photos and videos and send real time update to board control office. Ultrasonic sensors have been employed to ease the movement of robot with accuracy

Revised Manuscript Received on February 25, 2020.

* Correspondence Author

Prof. A Y. Prabhakar, E\&TC department, Bharati Vidyapeeth (deemed to be) University College of Engineering, Pune, India.

Akshit Sharma, E\&TC department, Bharati Vidyapeeth (deemed to be) University College of Engineering, Pune, India.

Ashish Kumar Singh, E\&TC department, Bharati Vidyapeeth (deemed to be) University College of Engineering, Pune, India.

Tushar Sharma, E\&TC department, Bharati Vidyapeeth (deemed to be) University College of Engineering, Pune, India.

(C) The Authors. Published by Blue Eyes Intelligence Engineering and Sciences Publication (BEIESP). This is an open access article under the CC BY-NC-ND license (http://creativecommons.org/licenses/by-nc-nd/4.0/) by avoiding obstacles. A robot can be controlled wirelessly. The wireless control provides ease of operation with additional benefits like lower installation cost. In the fast pacing world the internet automation is used for maneuver control and all other purposes like image or videos capture by the robot and shared via internet. Now-a-days the International border areas surveillance is a strenuous task. The guarding forces at border patrol with full seriousness yet surveillance of the whole area $24 \times 7$ is not possible. Safety of life at the same time securing of the areas are two different yet mandates. Primarily the aim is detection and tracking of a moving object via a camera mounted on the mobile robotic system and also to give the real time update to nearby board security control unit. The robotic system will act as a human replacement which could also be controlled manually by human itself on command. It would be helpful for the places at which humans cannot be sent for patrolling or there is a risk of life.

\section{SYSTEM OVERVIEW}

Our robot is designed for surveillance at international borders in a dedicated path .For traversing the path robot will be in automatic mode which will be using time stamp. The robot will detect any obstacles using ultrasonic sensors. It will send the notification to headquarter in case of intrusion detection and at the same time an alarm buzzer will be set on. As soon as the robot detect any obstacle at International border camera will turn on and live video feed using IOT will be sent directly to the Headquarter Office computer screen using an HTML page .After the intrusion or any obstacle is detected the robot can be operated manually and hence can track the trajectory of the Intruder in the respective territory.

Since International border is of great length it is necessary to deploy number of robots. To travel the path efficiently collaborative processing will be among the sensors has been used by establishing wireless sensor networks among the robot should be established to increase the efficiency of securing the border length. 


\section{BLOCK DIAGRAM}

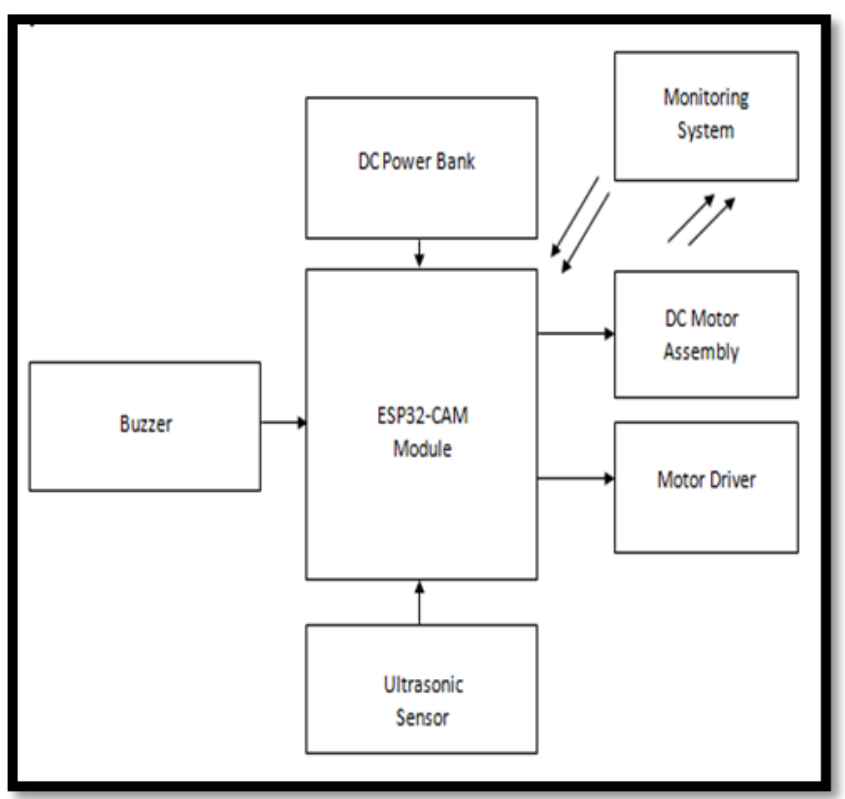

Figure 1: Block diagram

\section{HARDWARE IMPLEMENTATION}

\section{A. Ultrasonic Sensor}

Starting with the basic sensor used in the system that is the ultrasonic sensor. This device is used to measure the distance to an object using ultrasonic sound waves. It consists of a transducer that is used to send and receive ultrasonic pulses that relay back information about the proximity of an object. As a result, distinct echo patterns are produced due to the reflection of High-frequency sound waves from the boundaries. Ultrasonic sensors send a sound wave at a frequency above the range of human hearing. The sensor has a transducer that acts as a microphone to receive and send the ultrasonic sound. These sensors use a single transducer which sends a pulse and also receives the echo. Then the time lapse between the sending and receiving of the ultrasonic pulse is measured which determines the distance to a target. For example, an ultrasonic pulse is sent out at $40 \mathrm{KHz}$ which travels through the air and bounces back to the sensor if there is an obstacle or object. Thus the distance can be calculated through the time taken to travel and the speed of sound.

\section{B. Motor Driver Module L293D}

The Motor Driver module is used for Motors which helps us to control the working speed and direction of two motors simultaneously. It is designed and developed according to the L293D IC. This is because it helps in providing bidirectional drive currents at voltages ranging from $5 \mathrm{~V}$ to $36 \mathrm{~V}$. Also the L293D is a 16 pin IC, with eight pins on each side which helps in controlling the two DC motors simultaneously. Moreover, there are 4 INPUT and 4 OUTPUT pins and it also consists of 2 ENABLE pins for each motor. The rotation of the motors depends on these Enable pins. The L293D Adapter Board is designed in such a way that it can be used as a dual DC motor driver or bipolar stepper motor driver. It also has a separate logic supply which is required to reduce dissipation and further it also consists of the output clamping diodes which are used for protections. The L293D also has high noise immunity and over-temperature protection. It can also deliver output current up to $600 \mathrm{~mA}$ per channel.

\section{ESP32-CAM}

ESP32-CAM module consumes low power, is small in size and is based on ESP32. It has an onboard TF slot to connect the OV2640 camera to the module. This module supports both Wi-Fi and Bluetooth and has an onboard flash. It supports multiple sleep modes such as Deep sleep, Modern sleep and Light sleep with current as low as $6 \mathrm{~mA}, 20 \mathrm{~mA}$ and $6.7 \mathrm{~mA}$ respectively. Its dimensions are $40.5 \mathrm{~mm} \times 27 \mathrm{~mm} x$ $4.5 \mathrm{~mm}$. It is highly suited for IoT applications including face detection, wireless surveillance, uploading images and configuration with Message Queuing Telemetry Transport control of a device wirelessly. The board has 10 General Purpose Input Output pins, 3 Ground pins, 2 Power input pins and 1 Power output pin.

\section{SOFTWARE IMPLEMENTATION}

\section{A. Arduino IDE}

This application supports multiple computing platforms and supports codes written in $\mathrm{C}$ and $\mathrm{C}++$ programming language. It has an inbuilt software library which is helpful and can be reused for multiple similar input and output procedures. Code for the robotic system is written in this application which is then compiled to check for any errors. The code when once compiled successfully, with the use of USB to Transistor-Transistor Logic Serial converter is then uploaded on the ESP32-CAM module. This application also has a feature to support the third party hardware which can be added to its directory including the board definitions, core libraries, programmer definitions as well as the bootloaders. It also supports a serial monitor which is used for displaying the serial sent from the Arduino over USB or serial converter.

\section{B. C Programming Language}

The programming for the ESP32-Cam module is done in C language in Arduino IDE. Programming features such as recursion, lexical variable scope and structured programming are supported by it. The code written in this language are portable, more optimized than Assembly language and easier to maintain. This language was developed by Dennis Ritchie and Bell Labs and was first appeared in 1972. This language was developed to provide language constructs that provide efficient mapping to the instructions of the machine. This language is accessible by carious platforms ranging from embedded microcontrollers to the supercomputers.

\section{CloudMQTT}

Message Queuing Telemetry Transport is used to implement the IoT part of the robotic system. This employs the use of asynchronous communication protocol wherein the sender and the receiver don't interact simultaneously with the message queue. It is very easy to setup a Cloud MQTT instance starting with the creation of account and login to the control panel. The two main terms associated with it is Client and Broker. 
It works on the principle of Subscribe and Publish where the MQTT Client runs the library and establishes connection with the MQTT broker over a network and MQTT broker's function is to send the subscribed messages to intended client.

\section{Android Web Studio}

This application is used to support application development within the Android OS based on Gradle build system, an emulator, templates of code and is integrated with Github. It contains Android app modules, library modules and Google app engine modules and has an Instant Push feature for uploading the code and resource changes in the running application. It also has a code editor which assists in writing the code, assist in completion of code and analysis. This application was used in the prototype to create a console for manual tracking of the robotic system.

\section{RESULT}

The prototype successfully does surveillance of the given area wherein the live video streaming was seen via HTML page created using Arduino IDE for the ESP32-CAM module. The prototype uploads images to produce the live stream with minimum delay as well as is capable of face detection. To enable manual trigger, an android application is created where the console is present to control the movements of the robotic system. The snapshots of the working model are shown below.

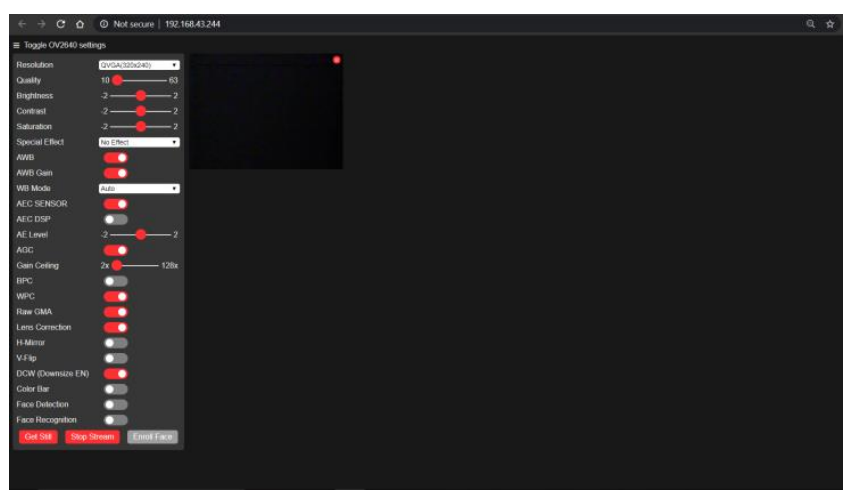

Figure 2: HTML web page which will be displaying the live feed

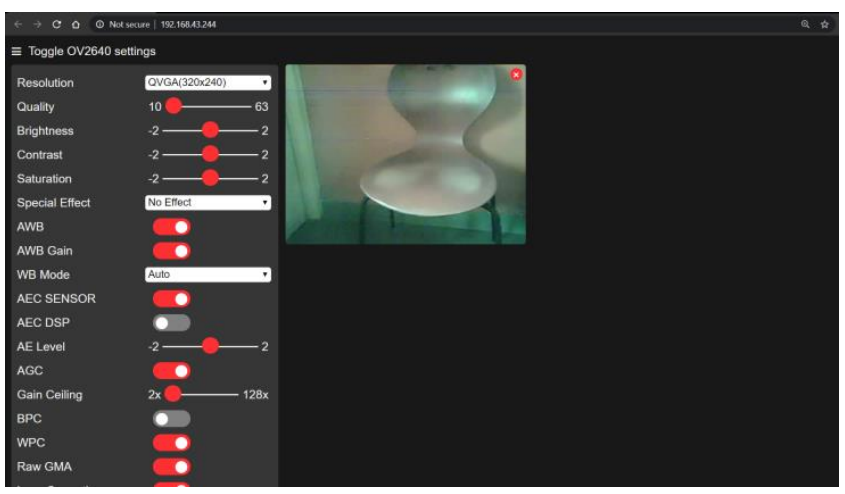

Figure 3: Still image of the live feed from the prototype

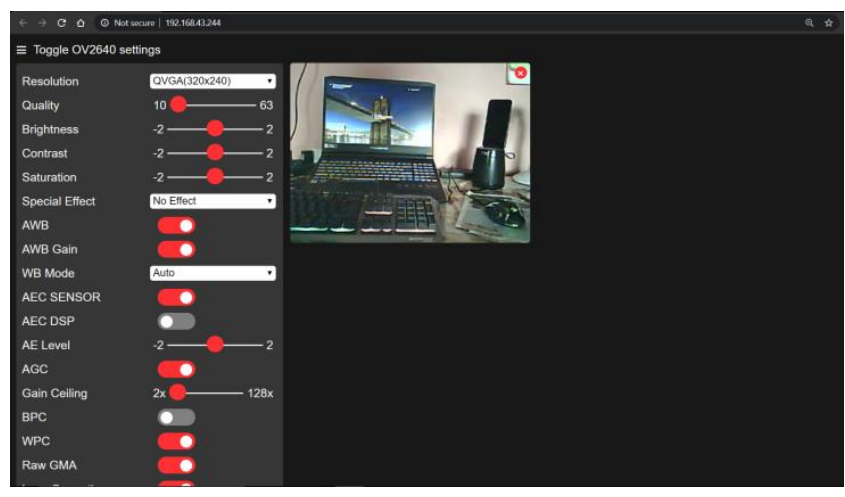

Figure 4: Still image of the live feed from the prototype

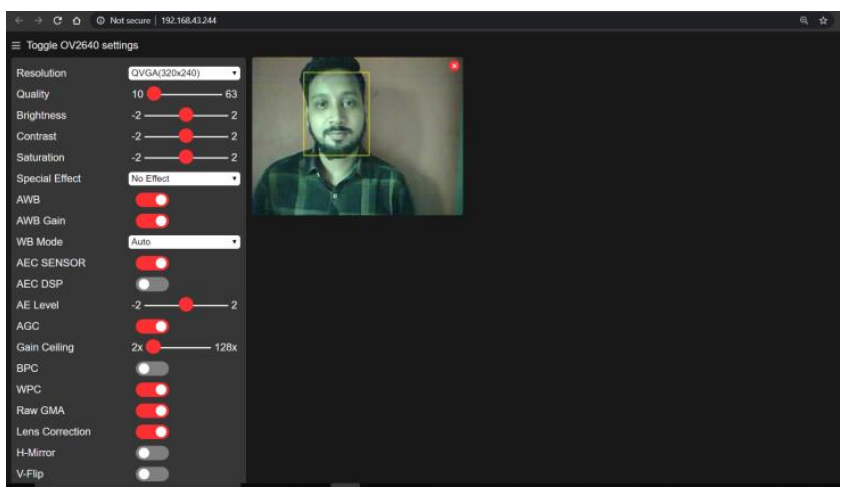

Figure 5: Still image showing face detection in real time

\section{$\mathrm{BZ}_{2}$. Still image showing face detection in real time}

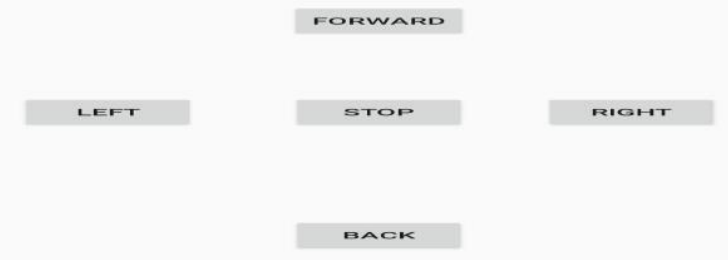

Figure 6: Android Application developed to control the robot manually

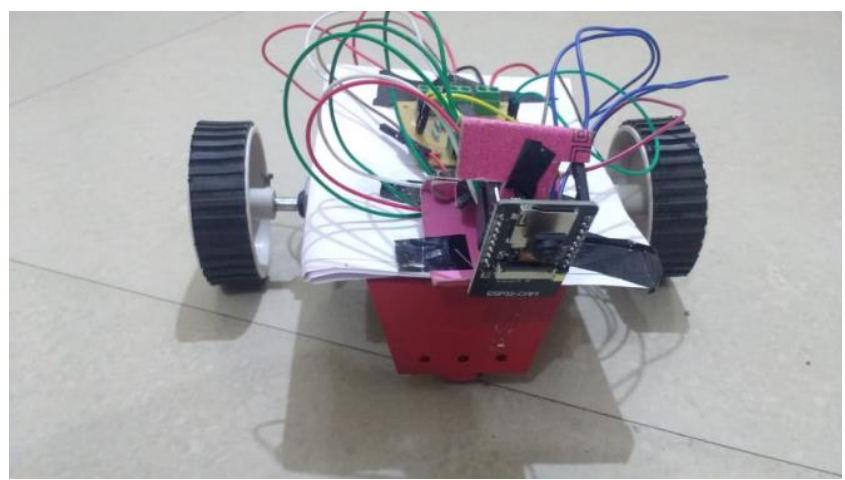

Figure 7: Prototype 


\section{CONCLUSION}

With the advancements in the field of technology, our model aims at providing better surveillance, security and thereby reducing the risk to human lives. In future applications, the prototype can be configured to the specifications of the area where it needs to be deployed such as tire, chassis, motor, camera and even the microcontroller to be used such that the robot works efficiently in the desired area. Multiple units need to be deployed to cover a given area efficiently to support collaborative processing. It is very cost friendly and provides good accuracy thereby proving to be a better tool to gather intelligence, automate the monitoring process and increase the overall effective security diameter. The constraint currently is the loss of connection between the robotic system and the network but for military purposes in future, it can be overcome when the system will be operating at the military bought bandwidths. Another constraint is the low cost camera used which can be configured specific to the area in which it needs to be used and thereby determining the type of camera to use to have a stable live feed as well as be capable of accurate detection of human presence. The working prototype can successfully do monitoring of a given plain area with live stream, detect human presence via face detection and also be manually controlled using the android application.

\section{REFERENCES}

1. Strategy Market Research Consulting, Internet of Robotic Things Global Market Outlook 2017-2023. Available: http://www.strategymrc.com/report/internet-of-robotic-thingsmarket-20 17.

2. IEEE Robotics \& Automation Society. Available: http://www.ieeeras.org/technical-committees/117-technicalcommittees/ networked-robots/146-networked-robots.

3. Implementation of Spy Robot for a surveillance system using Internet Protocol of Raspberry Pi. Available: https://www.researchgate.net/publication/317170129_Implementation_ of_Spy_Robot_for_A_Surveillance-

System_using_Internet_Protocol_of_Raspberry_Pi .

4. R. Roman, J. Zhou, and J. Lopez, "Applying Intrusion Detection Systems to Wireless Sensor Networks," in Consumer Communications and Networking Conference, 2006. CCNC 2006. 3rd IEEE, vol. 1, Jan 2006, pp. 640-644.

5. IEEE Robotics \& Automation Society. Available: http://www.ieeeras.org/technical-committees/117-technicalcommittees/ networked-robots/146-networked-robots.

6. Robotic Wireless Sensor Networks. Available: https://www.reseacrhgate.net/publication/316985507_Robotic_Wireles s_Sensor_Networks

7. A survey on MQTT: A Protocol of Internet of Things. Available: https://www.reseacrhgate.net/publication/316018571_A_SURVEY_O N_MQTT_A_PROTOCOL_OF_INTERNET_OF_THINGS.

8. Using the MQTT Protocol in Real Time for Synchronizing IoT Device State).Available: https://iajit.org/PDF/Special\%20Issue\%202018,\%20No.\%203A/17406. pdf

9. Apply Android Studio (SDK) Tools. Available: https://www.reseacrhgate.net/publication/331673953_Apply_Android Studio SDK Tools

10. Android Application Development using Android Studio. Available: http://www.ijcaonline.org/proceedings/rtfem2016/number1/25479-510 $\underline{9}$

\section{AUTHORS PROFILE}

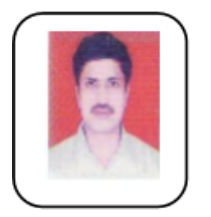

Prof. A Y. Prabhakar, Associate Professor Electronics and Telecommunication, M.Tech (pursuing PhD) Publication Details:

1. MICROWAVE ANTENNAS IN DEFENCE FOR ELECTRONIC WARFARE APPLICATION“by Hashpreet Singh, Amit Gupta, Sonali Dutta, A.

Prabhakar,International Journal of Electrical, Electronics and Data Communication -ISSN: 2320-2084 Volume-2, Issue-3, March-2014 pp: (112-116).

2. GSM BASED AGRICULTURE MONITORING SYSTEM by Aprajita Anand, Akansha Parasar A Prabhakar, International Research Journal of Engineering and Technology (IRJET) e-ISSN: 2395 -0056 Volume: 04 Issue: 03 | March -2017 www.irjet.net p-ISSN:2395-0072

3. Techno - Science revolution and justice delivery system by A. Prabhakar, Prof. Dr. A. Lakshminath Insights on global challenges and opportunities for the century ahead

Professional Memberships: Associate Member Institute of Electronics and Telecommunication Engineers Achievements: Bhartiyam Coordinator, Dept. T\&P Coordinator.

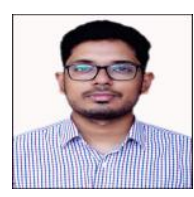

Tushar Sharma Student, B.Tech, Electronics \& Telecommunication

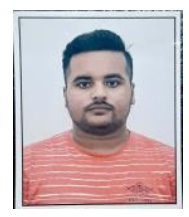

Akshit Sharma Student, B.Tech, Electronics \& Telecommunication

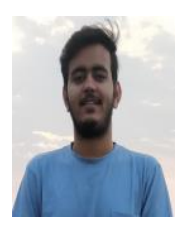

Ashish Kumar Singh, Student, B.Tech, Electronics \& Telecommunication 